\title{
A PE-MUSIC Algorithm for Sparse Array in MIMO Radar
}

\author{
Yucai Pang, Song Liu (iD, and Yun He \\ Chongqing University of Posts and Telecommunications, Chongqing 400065, China
}

Correspondence should be addressed to Song Liu; liusong@cqupt.edu.cn

Received 3 December 2020; Revised 4 January 2021; Accepted 20 January 2021; Published 4 February 2021

Academic Editor: Yun Bai

Copyright (c) 2021 Yucai Pang et al. This is an open access article distributed under the Creative Commons Attribution License, which permits unrestricted use, distribution, and reproduction in any medium, provided the original work is properly cited.

\begin{abstract}
Larger array aperture is provided by sparse arrays than uniform ones, which can improve the angle estimation resolution and reduce the cost of system evidently. However, manifold ambiguity is introduced due to the array sparsity. In this paper, a Power Estimation Multiple-Signal Classification (PE-MUSIC) algorithm is proposed to solve the manifold ambiguity of arbitrary sparse arrays for uncorrelated sources in Multiple-Input Multiple-Output (MIMO) radar. First, the paired direction of departure (DOD) and direction of arrival (DOA) are obtained for all targets by MUSIC algorithm, including the true and spurious ones; then, the well-known Davidon-Fletcher-Powell (DFP) algorithm is applied to estimate all targets' power values, among which the value of a spurious target trends to zero. Therefore, the ambiguity of sparse array in MIMO radar can be cleared. Simulation results verify the effectiveness and feasibility of the method.
\end{abstract}

\section{Introduction}

Multiple-Input Multiple-Output (MIMO) radar employs multiple transmit and receive elements and has the ability to plan transmissions and process received signals jointly. It has been the focus of research owing to its significant performance improvement compared to the conventional phased-array radar [1-3]. Sparse array MIMO radar has more degrees of freedom, larger effective array aperture, and more effective elements than uniform array MIMO radar [4-6]. Usually, larger effective array aperture produces more accurate direction of departure (DOD) and direction of arrival (DOA) estimation. Moreover, sparse sensor configuration can sharply reduce system cost and ease implementation.

However, array sparsity will cause ambiguity owing to the increased space between sensors. A lot of methods have been proposed to solve the ambiguity of conventional sparse arrays. Abramovich proposed a direct augmentation method which costs a high computational complexity [7-10]. A linear prediction approach was presented by Tufts but noise sensitive [11]. Vasylyshyn proposed a multiscale approach [12]. The main idea is using coarse estimation to disambiguate the fine estimation. However, the method is only suitable for some specific arrays. A virtual interpolation technique [13] was introduced to solve the ambiguity, but it is difficult to be applicable in practical applications. The power estimation approach was presented in lecture [14].

Few methods have been proposed to clear the ambiguity in sparse array MIMO radar. ESPRIT spectral search and ESPRIT polynomial root finding algorithm was presented in lecture [15], yet it is only applicable to the arrays with even number of transceiver elements. The idea of virtual interpolation was used for sparse array MIMO radar in [16]. Like the conventional sparse array, the same problem also exists. In [17], Alternating Least Squares (ALS) algorithm for tensor decomposition was developed to obtain the parameters of multitargets which is only suitable to the minimum redundant array. In $[18,19]$, the angle estimation algorithms were proposed for nested array and coprime array in MIMO radar. However, the method of [17-19] cannot be applicable to arbitrary sparse array.

In this paper, we present a method to solve the ambiguity of sparse array MIMO radar. It is separated into two steps by utilizing MUSIC and Davidon-Fletcher-Powell (DFP) algorithms, respectively. First, the paired DODs and DOAs of all targets, including real ones and spurious ones, are estimated by traditional MUSIC; thereafter, the power values of 
all targets are obtained through DFP algorithm by substituting the estimated DODs and DOAs into a predefined cost function. The power value of any spurious target is very small or trends to zero. Consequently, we can eliminate all the spurious targets and thus the ambiguity of the array is cleared.

The rest of the paper is arranged as follows. Section two presents the system model, and the algorithm is extensively explained by Section three. Detailed simulations are carried out in Section four to verify the method. Finally, we conclude the paper in Section five.

\section{System Model}

A bistatic MIMO radar system consists of a transmit array of $M$ antenna elements and a receive array of $N$ antenna elements, respectively. Both arrays are configured sparsely. The transmit elements are located at the position of $d_{m}^{t}(m=$ $1,2, \ldots, M)$ and the receive elements located at $d_{n}^{r}(n=1,2, \ldots, N)$ measured in half-wavelength unit with $d_{1}^{\mathrm{t}}=d_{1}^{\mathrm{r}}=0$ for convenience. The targets are assumed to be far-field. At the transmit site, $P$ different temporally orthogonal narrowband signals are emitted simultaneously with identical bandwidth and center frequency. There are $P$ noncoherent targets located in the same range bin. The DOD and DOA of the $p$ th target with respect to the transmit array normal and the receive array normal are denoted by $\theta_{p}$ and $\varphi_{p}$. The output of the entire matched filters at the receiver can be expressed as

$$
\mathbf{x}(t)=\mathbf{A}(\varphi, \theta) \mathbf{s}(t)+\mathbf{n}(t),
$$

the $M N \times 1$ noise vector $\mathbf{n}(t)$ is assumed to be independent, zero-mean complex Gaussian distribution. $A(\varphi, \theta)$ is the array manifold matrix, which can be expressed as

$$
A(\varphi, \theta)=\left[\mathbf{a}_{1}\left(\varphi_{1}, \theta_{1}\right), \ldots, \mathbf{a}_{P}\left(\varphi_{P}, \theta_{P}\right)\right]
$$

where $\mathbf{a}_{p}\left(\varphi_{p}, \theta_{p}\right)$ is the Kronecker product of the receive and the transmit steering vectors for the $p$ th target

$$
\mathbf{a}_{p}\left(\varphi_{p}, \theta_{p}\right)=\mathbf{a}_{r}\left(\varphi_{p}\right) \otimes \mathbf{a}_{t}\left(\theta_{p}\right),
$$

where $p=1, \ldots, P$ and

$$
\begin{gathered}
\mathbf{a}_{t}\left(\theta_{p}\right)=\left[1, e^{j 2 \pi\left(\left(d_{2}^{t} / \lambda\right) \cos \theta_{p}\right.}, \ldots, e^{j 2 \pi\left(d_{M}^{t} / \lambda\right) \cos \theta_{p}}\right]^{T}, \\
\mathbf{a}_{r}\left(\varphi_{p}\right)=\left[1, e^{j 2 \pi\left(d_{2}^{r} / \lambda\right) \cos \varphi_{p}}, \ldots, e^{j 2 \pi\left(d_{N}^{r} / \lambda\right) \cos \varphi_{p}}\right]^{T},
\end{gathered}
$$

where $(\bullet)^{T}$ is the transpose operation. The $M N \times 1$ vector $\mathbf{s}(t)$ consists of the phases and amplitudes of all the $P$ sources at time $t$. It is denoted as

$$
\begin{aligned}
s(t) & =\left[s_{1}(t), s_{2}(t), \ldots, s_{P}(t)\right]^{T}, \\
E\left[s(t) s(t)^{T}\right] & =\operatorname{diag}\left[q_{1}, q_{2}, \ldots q_{P}\right],
\end{aligned}
$$

where $E[\bullet]$ is the expectation operator and $q_{p}=E\left[\mathbf{s}_{p}^{2}(t)\right]$ is the power of the $p$ th source. The sources are independent, identically distributed Gaussian random variables with zero means.

\section{PE-MUSIC Algorithm}

Covariance matrix of $\mathbf{x}(t)$ can be calculated by

$$
\widehat{R}=\frac{1}{L \sum_{l=1}^{L} \mathbf{x}(t) \mathbf{x}^{H}(t)},
$$

where $L$ is the snapshot; $(\bullet)^{H}$ is the conjugate transpose operator. The MUSIC spectrum can be denoted as

$$
f_{\text {MUSIC }}=\frac{1}{\mathbf{a}^{H}(\varphi, \theta) \widehat{E}_{n} \widehat{E}_{n}^{H} \mathbf{a}(\varphi, \theta)},
$$

where $\widehat{E}_{n}$ is the estimation value of noise subspace, involving the eigenvectors corresponding to the last $M N-P$ eigenvalues by singular decomposition of $\widehat{R}$. According to (9), the paired DODs and DOAs of target can be obtained by twodimensional search.

Generally, $P+K$ sources will be obtained by traditional MUSIC method when a nontrivial ambiguity occurs for the abovementioned $P$ sources. Therefore, we can indicate $\left[\left(\varphi_{1}^{\prime}, \theta_{1}^{\prime}\right), \ldots,\left(\varphi_{P}^{\prime}, \theta_{P}^{\prime}\right), \ldots,\left(\varphi_{P+K}^{\prime}, \theta_{P+K}^{\prime}\right)\right]$ as the DODs and DOAs of the estimated targets and denote $Q^{\prime}=\left[q_{1}^{\prime}, \ldots, q_{1}^{\prime} \ldots, q_{P+K}^{\prime}\right]^{T}$ as the corresponding estimated power values.

The entry of the covariance of $\mathbf{x}(t)$ is

$$
\begin{aligned}
& E\left[x_{i}(t) x_{k}(t)\right]=E\left[\left(\sum_{m=1}^{M} \sum_{p=1}^{P} \alpha_{p} e^{j 2 \pi \tau\left(\varphi_{p}, \theta_{p}, i, m\right)}+\sum_{m=1}^{M} n_{i m}\right)\left(\sum_{m_{1}=1}^{M} \sum_{p_{1}=1}^{P} \alpha_{p_{1}} e^{j 2 \pi \tau\left(\varphi_{p_{1}}, \theta_{p_{1}}, k, m^{\prime}\right)}+\sum_{m_{\prime}=1}^{M} n_{k m^{\prime}}\right)\right] \\
& =\sum_{m=1}^{M} \sum_{p=1}^{P} e^{j 2 \pi\left[\tau\left(\varphi_{p}, \theta_{p}, i, m\right)+\tau\left(\varphi_{p}, \theta_{p}, k, m\right)\right]} q_{p}+\sum_{m=1}^{M} E\left[n_{i m} n_{k m}\right]
\end{aligned}
$$

where $i, k=1,2, \ldots, N$ and $\tau\left(\varphi_{p}, \theta_{p}, i, m\right)=\left(d_{m}^{t} / \lambda\right) \cos \theta_{p}+$ $\left(d_{i}^{r} / \lambda\right) \cos \varphi_{p}$.

$E\left[x_{i}(t) x_{k}(t)\right]$ can be estimated by averaging $x_{i}(t) x_{k}(t)$ over the total $N_{s}$ snapshots, i.e.,

$$
E\left[x_{i}(t) x_{k}(t)\right]=\left(\frac{1}{N_{s}}\right) \sum_{t=1}^{N_{s}} x_{i}(t) x_{k}(t)
$$




$$
f\left(\mathbf{q}^{\prime}\right)=\sum_{\substack{k \leq i \\ i=1}}^{N}\left\|\sum_{m=1}^{M} \sum_{p=1}^{P+K} e^{j 2 \pi\left[\tau\left(\varphi_{p}^{\prime} \theta_{p}^{\prime}, i, m\right)+\tau\left(\varphi_{p}^{\prime}, \theta_{p}^{\prime} k, m\right)\right]} q_{p}^{\prime}-E\left[x_{i}(t) x_{k}(t)\right]\right\|^{2} \text { s.t. } \mathbf{q}^{\prime}>=\mathbf{0} .
$$

Substituting equation (10) into (12), we get

$$
\begin{aligned}
& f\left(q^{\prime}\right)=\sum_{k \leq i}^{N}\left\|\sum_{m=1}^{M} \sum_{p=1}^{P+K} e^{j 2 \pi\left[\tau\left(\varphi_{p}^{\prime} \theta_{p}^{\prime}, i, m\right)+\tau^{\prime}\left(\varphi_{p}^{\prime} \theta_{p}^{\prime}, k, m\right)\right]} q_{p}^{\prime}-\sum_{m=1}^{M} \sum_{p=1}^{P} e^{j 2 \pi\left[\tau\left(\varphi_{p}, \theta_{p}, i, m\right)+\tau\left(\varphi_{p}, \theta_{p}, k, m\right)\right]} q_{p}-\sum_{m=1}^{M} E\left[N_{i m} N_{k m}\right]\right\|^{2} \\
& i=1 \\
& =\sum_{k \leq i}^{N} \sum_{m=1}^{M}\left\|\sum_{p=1}^{P} e^{j 2 \pi\left[\tau\left(\varphi_{p}^{\prime} \theta_{p}^{\prime}, i, m\right)+\tau\left(\varphi_{p}^{\prime} \theta_{p}^{\prime} k, m\right)\right]}\left(q_{p}^{\prime}-q_{p}\right)+\sum_{p=P+1}^{P+K} e^{j 2 \pi\left[\tau\left(\varphi_{p}^{\prime} \theta_{p}^{\prime}, i, m\right)+\tau\left(\varphi_{p}^{\prime} \theta_{p}^{\prime} k, m\right)\right]} q_{p}^{\prime}-E\left[N_{i m} N_{k m}\right]\right\|^{2} \\
& i=1 \\
& \leq \sum_{k \leq i}^{N}\left\{\sum_{m=1}^{M}\left\|\sum_{p=1}^{P} e^{j 2 \pi\left[\tau\left(\varphi_{p}^{\prime} \theta_{p}^{\prime} i, m\right)+\tau\left(\varphi_{p}^{\prime} \theta_{p}^{\prime} k, m\right)\right]}\left(q_{p}^{\prime}-q_{p}\right)\right\|^{2}+\sum_{m=1}^{M}\left\|\sum_{p=P+1}^{P+K} e^{j 2 \pi\left[\tau\left(\varphi_{p}^{\prime} \theta_{p}^{\prime}, n, m\right)+\tau\left(\varphi_{p}^{\prime} \theta_{p}^{\prime} r, m\right)\right]} q_{p}^{\prime}\right\|^{2}+\sum_{m=1}^{M}\left\|E\left[N_{i m} N_{k m}\right]\right\|^{2}\right\} .
\end{aligned}
$$

The necessary and sufficient condition for minimization of function $f\left(\mathbf{q}^{\prime}\right)$ in equation (13) is $q_{p}^{\prime}=q_{p}, \quad p=1,2, \ldots P, \quad$ and $\quad q_{p}^{\prime}=0, \quad p=P+1, \ldots$, $P+K$. The power values of the true targets are much larger than the power values of the spurious targets which must be zero theoretically. So, we can distinguish between the true targets and the spurious ones by estimating the power values of all targets.

The DFP algorithm [20] that is one of the quasi-Newton methods is applied to the optimization of equation (9). It is much faster and more efficient than the Newton method. DFP requires only the gradient, not the second derivatives of the cost function.

The PE-MUSIC algorithm to solve the ambiguity for a sparse array in MIMO radar can be summarized as below:

Step 1. Obtain the paired direction of DODs and DOAs for all targets by traditional MUSIC, including the true targets and the spurious ones.

Step 2. Substitute all the paired DODs and DOAs estimated by Step 1 into the cost function equation (9) to estimate the power values related to all targets through the DFP algorithm. The estimated power value of the spurious target trends to zero. So, we can distinguish between the true target and the spurious target.

\section{Simulation}

Simulations have been done to verify the feasibility and effectiveness of the proposed method.

Simulation 1. The transmit array is a sparse array located at $\left[\begin{array}{lll}0 & 1 & 2\end{array}\right]$; the receive array is also a sparse array located at [0 2
$46]$. There is 1 source with the DOD and DOA as (28.67, $69.45^{\circ}$ ). The number of snapshots is 100 . Set the power of the source as $10 \mathrm{~dB}$.

Figure 1 illustrates the result of Simulation 1; the horizontal axis represents the DOA of a target, and the vertical axis depicts both DOD and power value of the target. In Figure 1, the solid squares are the paired DODs and DOAs of the targets; the circles refer to the corresponding estimated power values of the targets. As Figure 1 shows, there are a total of two targets estimated: $\left(28.67^{\circ}, 69.45^{\circ}\right)$ and $\left(28.67^{\circ}\right.$, $\left.130.47^{\circ}\right)$. The true target has been obtained by MUSIC algorithm correctly, but spurious one also appears due to the manifold ambiguity. It is difficult to distinguish between the true target and the spurious one by MUSIC algorithm. Substituting the estimated angle value of the two targets into equation (12), we can get the power values of all the targets as shown in Table 1 through DFP algorithm. From Table 1, we can know that $\left(28.67^{\circ}, 69.45^{\circ}\right)$ is the true target and another one is the spurious one. Therefore, we conclude that there are $P=1$ true target and 1 spurious target. The effectiveness of the proposed method has been verified by Simulation 1 where there is only one spurious target. More than one spurious target will be studied in Simulations 2 and 3 .

Simulation 2. The transmit array is a sparse array located at [0 $\left.\begin{array}{lllll}0 & 4 & 6 & 12\end{array}\right]$; the receive array is also a sparse array located at [0 $\left.\begin{array}{lll}0 & 4 & 9\end{array}\right]$. There are 3 sources with the DODs and DOAs as $\left(10.12^{\circ}, 15.23^{\circ}\right),\left(70.32^{\circ}, 25.28^{\circ}\right)$, and $\left(100.56^{\circ}, 110.22^{\circ}\right)$, respectively. The number of snapshots is 100 . Set the power of all sources as $10 \mathrm{~dB}$.

As what has been shown in Figure 2, the horizontal axis represents the DOD of a target, and the vertical axis depicts 


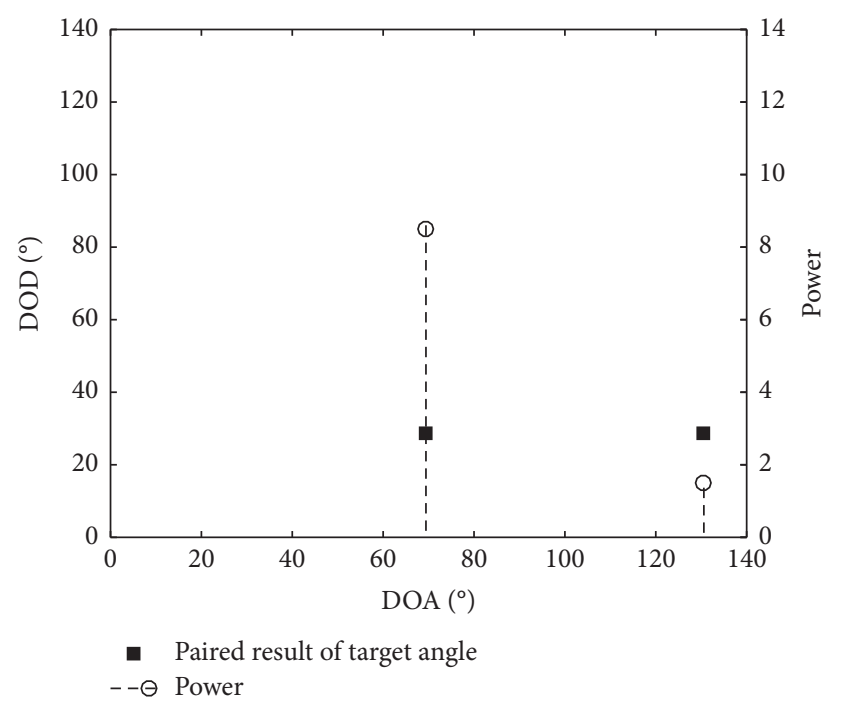

FIGURE 1: The result of Simulation 1.

TABle 1: The data of Simulation 1.

\begin{tabular}{lcc}
\hline DOD and DOA & Estimated power & Result \\
\hline$\left(28.67^{\circ}, 69.45^{\circ}\right)$ & $9.3 \mathrm{~dB}$ & True \\
$\left(28.67^{\circ}, 130.47^{\circ}\right)$ & $1.75 \mathrm{~dB}$ & Spurious \\
\hline
\end{tabular}

both DOA and power value of the target. It denotes that there are a total of six targets estimated: $\left(10.12^{\circ}, 15.23^{\circ}\right)$, $\left(90.9^{\circ}, 15.23^{\circ}\right),\left(70.32^{\circ}, 25.28^{\circ}\right),\left(131.56^{\circ}, 25.28^{\circ}\right),\left(35.25^{\circ}\right.$, $\left.110.22^{\circ}\right)$, and $\left(100.56^{\circ}, 110.22^{\circ}\right)$. All the true targets and the spurious ones are obtained by MUSIC algorithm. Adopting the proposed method, we can get the power values of all the targets as Table 2 . By comparing the estimated power value of all targets, we can know that $\left(10.12^{\circ}, 15.23^{\circ}\right),\left(70.32^{\circ}\right.$, $\left.25.28^{\circ}\right)$, and $\left(100.56^{\circ}, 110.22^{\circ}\right)$ are the true targets and the remaining three are spurious ones. We can draw the conclusion that there are $P=3$ true targets and 3 spurious ones.

Simulation 3. The sparse transmit array is located at $\left[\begin{array}{llll}0 & 1 & 2 & 3\end{array}\right.$

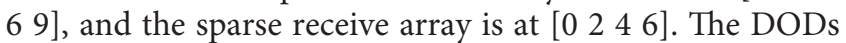
and DOAs of the true targets are $\left(18.26^{\circ}, 25.32^{\circ}\right),\left(35.18^{\circ}\right.$, $\left.60.69^{\circ}\right),\left(72.38^{\circ}, 15.36^{\circ}\right),\left(120.78^{\circ}, 100.58^{\circ}\right)$, and $\left(150.35^{\circ}\right.$, $\left.45.56^{\circ}\right)$. The number of snapshots is also 100 . The power values of all sources are $10 \mathrm{~dB}$ as well.

As Figure 3 indicates, there are a total of ten targets obtained by MUSIC algorithm. According to our proposed method, we can get the estimated power value as Table 3. From Table 3, we can know that $\left(72.38^{\circ}, 15.36^{\circ}\right),\left(18.26^{\circ}\right.$, $\left.25.32^{\circ}\right),\left(150.35^{\circ}, 45.56^{\circ}\right),\left(35.18^{\circ}, 60.69^{\circ}\right)$, and $\left(120.78^{\circ}\right.$, $\left.100.58^{\circ}\right)$ are the true targets; and $\left(120.78^{\circ}, 35.28^{\circ}\right),\left(72.38^{\circ}\right.$, $\left.92.06^{\circ}\right),\left(18.26^{\circ}, 95.52^{\circ}\right),\left(150.35^{\circ}, 107.46^{\circ}\right)$, and $\left(35.18^{\circ}\right.$, $\left.120.70^{\circ}\right)$ are the spurious ones. It is shown that the power values of the five true targets are much larger than the other five spurious ones. Therefore, the ambiguities can be easily cleared from all the targets obtained by MUSIC algorithm.

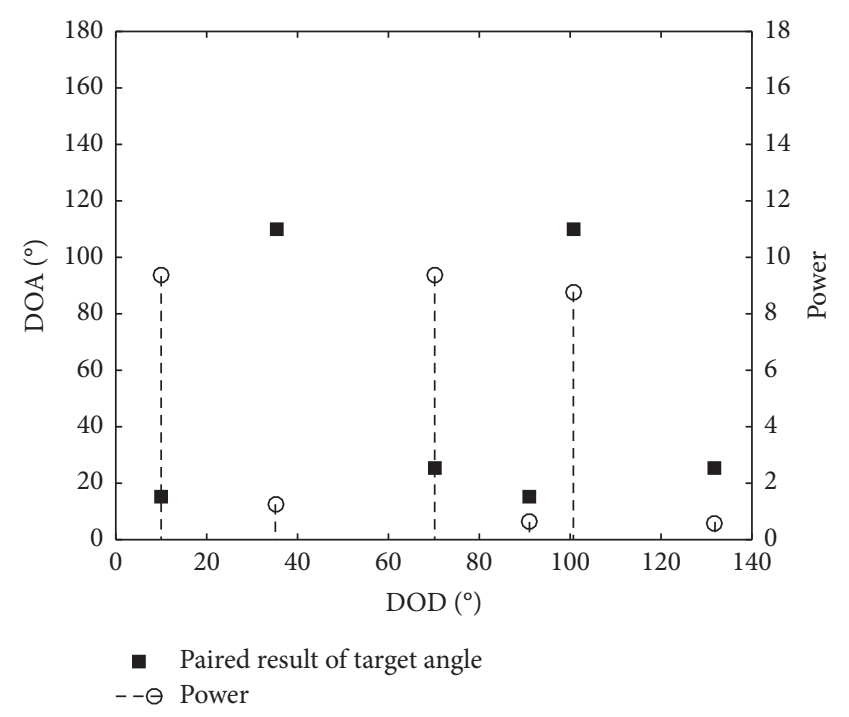

Figure 2: The result of Simulation 2.

TAble 2: The data of Simulation 2.

\begin{tabular}{lcc}
\hline DOD and DOA & Estimated power & Result \\
\hline$\left(10.12^{\circ}, 15.23^{\circ}\right)$ & $9.72 \mathrm{~dB}$ & True \\
$\left(90.9^{\circ}, 15.23^{\circ}\right)$ & $-1.98 \mathrm{~dB}$ & Spurious \\
$\left(70.32^{\circ}, 25.28^{\circ}\right)$ & $9.73 \mathrm{~dB}$ & True \\
$\left(131.56^{\circ}, 25.28^{\circ}\right)$ & $-2.23 \mathrm{~dB}$ & Spurious \\
$\left(35.25^{\circ}, 110.22^{\circ}\right)$ & $1 \mathrm{~dB}$ & Spurious \\
$\left(100.56^{\circ}, 110.22^{\circ}\right)$ & $9.42 \mathrm{~dB}$ & True \\
\hline
\end{tabular}

Simulation 4. Set the power of the source as $3.4 \mathrm{~dB}$. Other simulation parameters are the same as in Simulation 1.

As Figure 4 and Table 4 show, we can know that the estimated accuracy of power is decreased compared with Simulation 1 when the power of the source is small. But we can also distinguish between the true target and the spurious one through our proposed method.

Simulation 5. Set the power of the source differently. The power of the first source is $12.39 \mathrm{~dB}$, the power of the second source is $9 \mathrm{~dB}$, and the power of the third source is $7.74 \mathrm{~dB}$. Other simulation parameters are the same as in Simulation 2.

From Figure 5 and Table 5 , we can get that $\left(10.12^{\circ}\right.$, $\left.15.23^{\circ}\right),\left(70.32^{\circ}, 25.28^{\circ}\right)$, and $\left(100.56^{\circ}, 110.22^{\circ}\right)$ are the true targets; $\left(90.9^{\circ}, 15.23^{\circ}\right),\left(131.56^{\circ}, 25.28^{\circ}\right)$, and $\left(35.25^{\circ}, 110.22^{\circ}\right)$ are the spurious targets. The result is the same as in Simulation 2. It is shown that the proposed method still works well when the power of every source is different.

Simulation 6. Set the power of the source differently. The power of the first source is $11.7 \mathrm{~dB}$, the power of the second source is $10 \mathrm{~dB}$, the power of the third source is $8.1 \mathrm{~dB}$, the power of the fourth source is $7.7 \mathrm{~dB}$, and the power of the fifth source is $9.5 \mathrm{~dB}$. Other simulation parameters are the same as in Simulation 3. 


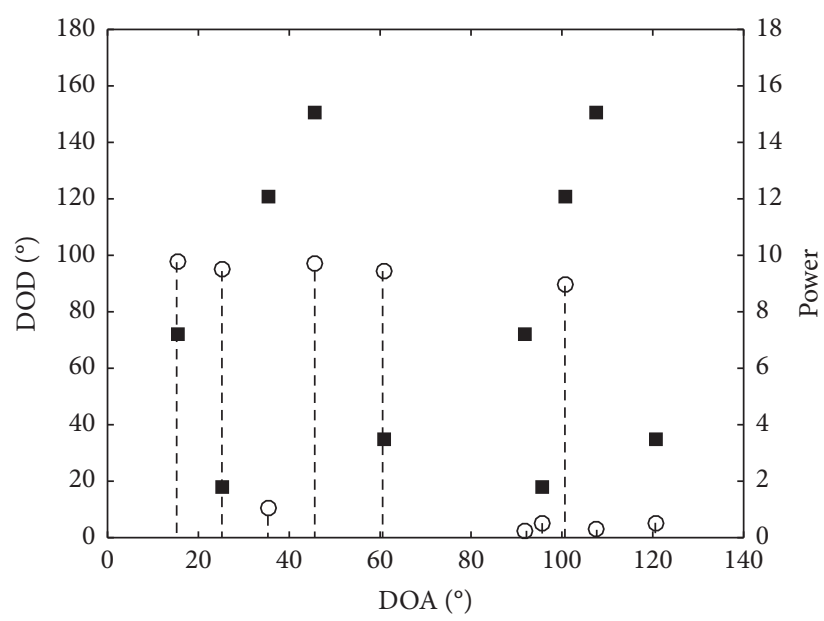

- Paired result of target angle $--\Theta$ Power

Figure 3: The result of Simulation 3.

TABle 3: The data of Simulation 3.

\begin{tabular}{lcc}
\hline DOD and DOA & Estimated power & Result \\
\hline$\left(72.38^{\circ}, 15.36^{\circ}\right)$ & $9.89 \mathrm{~dB}$ & True \\
$\left(18.26^{\circ}, 25.32^{\circ}\right)$ & $9.77 \mathrm{~dB}$ & True \\
$\left(120.78^{\circ}, 35.28^{\circ}\right)$ & $0.21 \mathrm{~dB}$ & Spurious \\
$\left(150.35^{\circ}, 45.56^{\circ}\right)$ & $9.88 \mathrm{~dB}$ & True \\
$\left(35.18^{\circ}, 60.69^{\circ}\right)$ & $9.76 \mathrm{~dB}$ & True \\
$\left(72.38^{\circ}, 92.06^{\circ}\right)$ & $-6.15 \mathrm{~dB}$ & Spurious \\
$\left(18.26^{\circ}, 95.52^{\circ}\right)$ & $-2.88 \mathrm{~dB}$ & Spurious \\
$\left(120.78^{\circ}, 100.58^{\circ}\right)$ & $9.52 \mathrm{~dB}$ & True \\
$\left(150.35^{\circ}, 107.46^{\circ}\right)$ & $-5.56 \mathrm{~dB}$ & Spurious \\
$\left(35.18^{\circ}, 120.70^{\circ}\right)$ & $-2.78 \mathrm{~dB}$ & Spurious \\
\hline
\end{tabular}

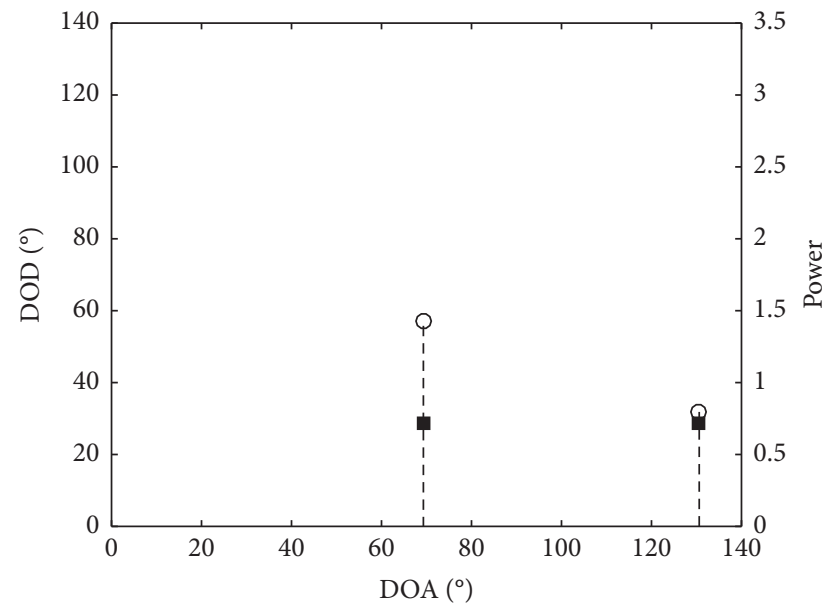

- Paired result of target angle $--\Theta$ Power

Figure 4: The result of Simulation 4.
TABLE 4: The data of Simulation 4.

\begin{tabular}{lcc}
\hline DOD and DOA & Estimated power & Result \\
\hline$\left(28.67^{\circ}, 69.45^{\circ}\right)$ & $1.56 \mathrm{~dB}$ & True \\
$\left(28.67^{\circ}, 130.47^{\circ}\right)$ & $-1 \mathrm{~dB}$ & Spurious \\
\hline
\end{tabular}

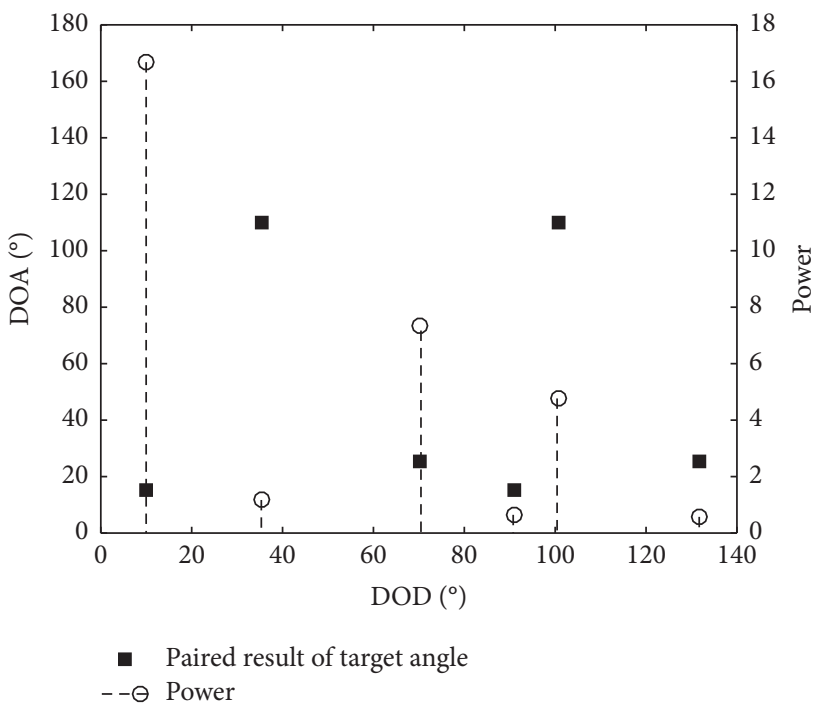

FIgURE 5: The result of Simulation 5.

Table 5: The data of Simulation 5.

\begin{tabular}{lcc}
\hline DOD and DOA & Estimated power & Result \\
\hline$\left(10.12^{\circ}, 15.23^{\circ}\right)$ & $12.22 \mathrm{~dB}$ & True \\
$\left(90.9^{\circ}, 15.23^{\circ}\right)$ & $-1.9 \mathrm{~dB}$ & Spurious \\
$\left(70.32^{\circ}, 25.28^{\circ}\right)$ & $8.66 \mathrm{~dB}$ & True \\
$\left(131.56^{\circ}, 25.28^{\circ}\right)$ & $-2.4 \mathrm{~dB}$ & Spurious \\
$\left(35.25^{\circ}, 110.22^{\circ}\right)$ & $0.69 \mathrm{~dB}$ & Spurious \\
$\left(100.56^{\circ}, 110.22^{\circ}\right)$ & $6.78 \mathrm{~dB}$ & True \\
\hline
\end{tabular}

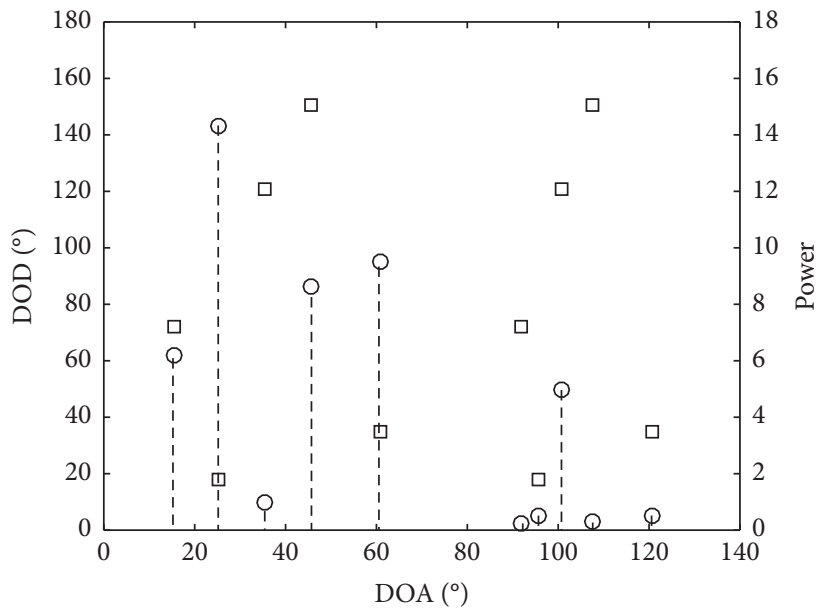

Paired result of target angle

- $\ominus$ Power

FIgURE 6: The result of Simulation 6. 
TABLE 6: The data of Simulation 6.

\begin{tabular}{lcc}
\hline DOD and DOA & Estimated power & Result \\
\hline$\left(72.38^{\circ}, 15.36^{\circ}\right)$ & $7.92 \mathrm{~dB}$ & True \\
$\left(18.26^{\circ}, 25.32^{\circ}\right)$ & $11.56 \mathrm{~dB}$ & True \\
$\left(120.78^{\circ}, 35.28^{\circ}\right)$ & $-0.1 \mathrm{~dB}$ & Spurious \\
$\left(150.35^{\circ}, 45.56^{\circ}\right)$ & $9.36 \mathrm{~dB}$ & True \\
$\left(35.18^{\circ}, 60.69^{\circ}\right)$ & $9.77 \mathrm{~dB}$ & True \\
$\left(72.38^{\circ}, 92.06^{\circ}\right)$ & $-6.19 \mathrm{~dB}$ & Spurious \\
$\left(18.26^{\circ}, 95.52^{\circ}\right)$ & $-2.87 \mathrm{~dB}$ & Spurious \\
$\left(120.78^{\circ}, 100.58^{\circ}\right)$ & $6.96 \mathrm{~dB}$ & True \\
$\left(150.35^{\circ}, 107.46^{\circ}\right)$ & $-5.66 \mathrm{~dB}$ & Spurious \\
$\left(35.18^{\circ}, 120.70^{\circ}\right)$ & $-2.85 \mathrm{~dB}$ & Spurious \\
\hline
\end{tabular}

As what have been shown in Figure 6 and Table 6, the true targets are $\left(72.38^{\circ}, 15.36^{\circ}\right),\left(18.26^{\circ}, 25.32^{\circ}\right),\left(150.35^{\circ}\right.$, $\left.45.56^{\circ}\right),\left(35.18^{\circ}, 60.69^{\circ}\right)$, and $\left(120.78^{\circ}, 100.58^{\circ}\right)$; the others are the spurious targets. The result is the same as in Simulation 3. It is demonstrated that the proposed method still performs well when the number of targets is more than the number in Simulation 5 and the power of every source is different.

\section{Conclusion}

Aiming at solving the manifold ambiguities in MIMO radar, a new PE-MUSIC algorithm has been proposed in this paper. In order to eliminate the spurious targets, a new cost function is used to estimate the power values of all targets obtained by MUSIC. The extra computation expense brought by the DFP algorithm is much trivial. From the simulation results, it is easy to clear the ambiguities or spurious targets from all the estimates by the proposed method. Different kinds of arrays, different-source power values, and different number of targets are analyzed in the simulations. The feasibility and the effectiveness of the proposed method are verified.

\section{Data Availability}

No data were used to support this study.

\section{Conflicts of Interest}

The authors declare that they have no conflicts of interest regarding the publication of this paper.

\section{Acknowledgments}

This study was supported by the Natural Science Foundation of Chongqing Municipal Education Commission, China (nos. KJQN201800631 and KJQN201800618) and the Natural Science Foundation of Chongqing, China (cstc2020jcyjmsxm0909).

\section{References}

[1] E. Fishler, A. Haimovich, R. Blum, D. Chizhik, L. Cimini, and R. Valenzuela, "MIMO radar: an idea whose time has come," in Proceedings of the IEEE Radar Conference, pp. 71-78, Philadelphia, Pa, USA, March 2004.
[2] A. Haimovich, R. Blum, and L. Cimini, "MIMO radar with widely separated antennas," IEEE Signal Processing Magazine, vol. 25, no. 1, pp. 116-129, 2008.

[3] J. Li and P. Stoica, "MIMO radar with colocated antennas," IEEE Signal Processing Magazine, vol. 24, no. 5, pp. 106-114, 2007.

[4] E. Fishler, A. Haimovich, R. S. Blum, L. J. Cimini, D. Chizhik, and R. A. Valenzuela, "Spatial diversity in radars-models and detection performance," IEEE Transactions on Signal Processing, vol. 54, no. 3, pp. 823-838, 2006.

[5] J. Li, P. Stoica, L. Xu, and W. Roberts, "On parameter identifiability of MIMO radar," IEEE Signal Processing Letters, vol. 14, no. 12, pp. 968-971, 2007.

[6] L. Xu, J. Li, and P. Stoica, "Target detection and parameter estimation for MIMO radar systems," IEEE Transactions on Aerospace and Electronic Systems, vol. 44, no. 3, pp. 927-939, 2008.

[7] Y. I. Abramovich, V. G. Gaitsgory, and N. K. Spencer, "Stability of Manifold ambiguityresolution in DOA estimation with non-uniform linear antenna arrays," International Conference on Acoustics, Speech, and Signal Processing, vol. 5, pp. 3117-3120, 2000.

[8] Y. I. Abramovich, N. K. Spencer, and A. Y. Gorokhov, "DOA estimation for noninteger linear antenna arrays with more uncorrelated sources than sensors," IEEE Transactions on Signal Processing, vol. 48, no. 4, pp. 943-955, 2000.

[9] Y. I. Abramovich, N. K. Spencer, and A. Y. Gorokhov, "Positive-definite Toeplitz completion in DOA estimation for nonuniform linear antenna arrays. II. Partially augmentable arrays," IEEE Transactions on Signal Processing, vol. 47, no. 6 , pp. 1502-1521, 1999.

[10] Y. I. Abramovich, N. K. Spencer, and A. Y. Gorokhov, "Resolving manifold ambiguities in direction-of-arrival estimation for nonuniform linear antenna arrays," IEEE Transactions on Signal Processing, vol. 47, no. 10, pp. 2629-2643, 1999.

[11] D. W. Tufts and R. K. HongyaGe, "Resolving ambiguities in estimation spatial frequencies in sparse linear array," Proceedings of the International Conference on Acoustics, Speech, and Signal Processing, vol. 2, pp. 345-348, 1994.

[12] V. I. Vasylyshyn, "Direction of arrival estimation using ESPRIT with sparse arrays," in Proceedings of the 6th European Radar Conference, pp. 246-249, Rome, Italy, April 2009.

[13] X. Shi, "A new approach to estimate DOA using virtual interpolation technique," in Proceedings of the 9th International Conference on ICSP, pp. 179-182, Beijing, China, October 2008.

[14] Z. He, Z. Zhao, Z. Nie, P. Tang, J. Wang, and Q.-H. Liu, "Method of solving ambiguity for sparse array via power estimation based on MUSIC algorithm," Signal Processing, vol. 92, no. 2, pp. 542-546, 2012.

[15] J. Zhang and X. Peilei, "Joint DOD and DOA estimation for bistatic MIMO radar," Systems Engineering and Electronics, vol. 31, no. 11, pp. 2268-2272, 2010.

[16] W. Wang, X.-P. Wang, and L. I. Xin, "Multi-target localization for non-uniform linear array of MIMO radar," Systems Engineering and Electronics, vol. 34, no. 3, pp. 477-481, 2012.

[17] J. Fan, H. Gu, W. Su, and D. Gong, "Angle and Doppler frequency estimation based on non-uniform MIMO radar system," in Proceedings of the 2015 IEEE International Conference on Signal Processing, Communications and Computing (ICSPCC), Zhejiang, China, September 2015.

[18] C. Wang, Z. Li, and X. Zhang, "FDA-MIMO for joint angle and range estimation: unfolded coprime framework and 
parameter estimation algorithm," IET Radar, Sonar \& Navigation, vol. 14, no. 6, pp. 917-926, 2020.

[19] Z. Fu, P. Charge, and Y. Wang, "A virtual nested MIMO array exploiting fourth order difference coarray," IEEE Signal Processing Letters, vol. 27, pp. 1140-1144, 2020.

[20] D. G. Leunberger and Y. Ye, Linear and Non-linear Programming, Springer, Berlin, Germany, 2008. 